\title{
Do patients with neutropenic sepsis need granulocyte stimulating factor (GCSF)?
}

\author{
Authors: Thomas Coventry and Faisal Azam
}

\section{Aims}

We performed this review to assess the prescribing practice of therapeutic granulocyte stimulating factor (GCSF) in patients admitted with neutropenic sepsis (NS) after systemic anticancer therapy (SACT) at 'local hospital'.

NS is defined as neutrophil count of $\leq 0.5$ and temperature of $\geq 38^{\circ} \mathrm{C}$. Therapeutic GCSF in the treatment of NS has a limited role. ASCO (American Society of Clinical Oncology), EORTC (European Organisation for Research and Treatment of Cancer) and NICE (National Institute for Health and Care Excellence) guidelines recommend it for patients with febrile neutropenia and high risk of infection-associated complications.

\section{Methods}

Retrospective analysis of case notes of patients, admitted with NS from January to December 2012, after treatment with SACT for solid tumours, was performed.

Indications defined by the guidelines are:

$>$ Fever $\geq 38^{\circ} \mathrm{C}+$ neutrophil count $<0.1 \mathrm{~g} / \mathrm{L}$ after SACT

AND at least one of the following:

$>$ aim of the of SACT (palliative for progressive disease)

$>$ hypotension on admission

$>$ clinical or radiological signs of pneumonia

$>$ duration of neutropenia ( $>10$ days)

$>$ age ( $>65$ years)

$>$ organ dysfunction/failure

proven invasive fungal infection.

\section{Results}

Total numbers of patients admitted with NS were 54.43 (80\%) patients were female. Mean age was 58 years. Breast cancer was the commonest tumour, with 23 (42\%) patients followed by lung cancer $(\mathrm{n}=7,13 \%)$. Mean neutrophil count on admission was $0.61 \mathrm{~g} / \mathrm{L}$ (range: $0.01-3.1 \mathrm{~g} / \mathrm{L}$ ). Average length of stay was 6 days (1-24). $42(77 \%)$ patients had a temperature of $<37.5^{\circ} \mathrm{C}$ on admission and $23(43 \%)$ patients had a neutrophil count of $>0.5 \mathrm{~g} / \mathrm{L}$.
GCSF was administered in $46(94 \%)$ patients. Nine patients were already on prophylactic GCSF at the time of admission, while $37(68 \%)$ were started in the hospital with a therapeutic intention.

Among the 37 patients who received therapeutic GCSF, it was only indicated in four (12\%) patients as per guidelines. The other $33(88 \%)$ patients had therapeutic GCSF with no indications. Median number of days on GCSF were 3 (range 1-7) and total number of GCSF injections were 124.97 out of 124 GCSF injections were administered without any defined indications.

\section{Conclusions}

Most of the therapeutic GCSF prescriptions were not evidence based. It was indicated and administered appropriately in 12\% of patients. Among the patients who received therapeutic GCSF for the treatment of NS, $88 \%$ were not indicated.

GCSF has a limited role in the treatment of SACT-induced NS. Appropriate prescribing will prevent unnecessary side effects, cost and nurse/pharmacy time. 